\title{
Effects of Secondary Flow on the Electromagnetic Separation of Inclusions from Aluminum Melt in a Square Channel by a Solenoid
}

\author{
Da SHU, Baode SUN, Ke LI, Jun WANG and Yaohe ZHOU \\ School of Materials Science and Engineering, Shanghai Jiao Tong University, Shanghai 200030 China. \\ E-mail: dshu@sjtu.edu.cn
}

(Received on May 17, 2002; accepted in final form on July 9, 2002)

\begin{abstract}
Effects of secondary flow of the melt on the separation of inclusions from molten aluminum flowing in a square channel by a solenoid were investigated theoretically and experimentally. Numerical methods were used to calculate the secondary flow, the separation efficiency of inclusions, and the particle motion. It is found that there appear two recirculating loop flows in a quarter cross section of the channel and the separation efficiency of inclusions is significantly improved by the secondary flow mainly owing to the mixing effect. The separation efficiency increases with the increase of the effective magnetic flux density and the frequency of magnetic field, and decreases significantly with the increase of the size of the separator channel for a constant value of $a / \delta$. However, it is possible to achieve high separation efficiency by using largesized square channels and high frequency magnetic field with the help of the mixing effect of secondary flow. The computed results of particle trajectories show that the secondary flow accelerates the transportation of the particles from the inner region to the vicinity of the wall and greatly shortens the separation time of those particles. The effects of secondary flow on the separation efficiency were confirmed by comparing the measured separation efficiency with the computed results.
\end{abstract}

KEY WORDS: inclusions; aluminum; electromagnetic separation; solenoid; secondary flow; separation efficiency; trajectory.

\section{Introduction}

Electromagnetic separation of non-metallic inclusions from aluminum melt is an emerging technology for the production of ultra-clean aluminum alloys to meet the growing demand for cleaner metals. It can, in principle, remove even micrometer-sized inclusions at almost constant rate by using high-intensity force fields. ${ }^{1,2)}$ The main advantage of electromagnetic separation is that the electromagnetic expulsive force exerted on inclusion particles is only dependent on the difference in electrical conductivity between the inclusions and melt and independent of the density, chemical composition, or different phases (solid, liquid or gas) of the inclusions. ${ }^{3,4)}$ Another advantage is that it offers very clean processes in view of environmental protection, as the use of fluxes, generally chlorides or fluorides, is avoided. Moreover, the melt is also protected against contamination if induction technology is utilized, since there is no contact between the melt and the power source due to the intrinsic properties of the magnetic fields. ${ }^{5}$

The principle of electromagnetic separation was proposed long ago, ${ }^{6)}$ and various schemes by exerting different types of electromagnetic field, including DC electric field with a crossed steady magnetic field, ${ }^{7)} \mathrm{AC}$ electric field, ${ }^{8)}$ stationary AC magnetic field, ${ }^{9)}$ traveling magnetic field, ${ }^{10)}$ simultaneous imposition of $\mathrm{AC}$ current and $\mathrm{AC}$ magnetic field, ${ }^{11)}$ and more recently, strong magnetic field created by a superconducting DC coil ${ }^{12)}$ were also presented. While the effectiveness and potential of this innovative method have been clearly demonstrated by those research works, its implementation in industrial applications under continuous flow conditions is scarcely reported. The main problem, at present, is that large and homogeneous electromagnetic force densities in large volumes are difficult to achieve, ${ }^{13)}$ which is especially true for the AC fields due to the wellknown skin effect. As a result, the induced flow of the melt by the unevenly distributed force field may appear, which is generally considered as extremely detrimental to the separation process and may render the effective separation of inclusions impossible. ${ }^{7,12,13)}$

El-Kaddah ${ }^{14)}$ calculated the particle motion in the molten pool of the ORNL electromagnetic separation process by an AC magnetic field and found that the vigorously stirred melt may cause some problems with the separation of small inclusions due to the entrapment of the inclusion particles within flow eddies. Taniguchi and Brimacombe ${ }^{8)}$ investigated the separation of inclusions from liquid metal flowing in a square pipe by an $\mathrm{AC}$ electric field and calculated the particle removal efficiency based on a concentration method by taking the secondary flows into account. The calculated re- 
sults of the particle removal efficiency, however, indicate that the secondary flows may transport particles from the inner region of the pipe to the vicinity of the wall where the electromagnetic pinch force is strong and thus increase the inclusion removal efficiency. Therefore, understanding of the role of the induced flow is central to the separation of inclusion particles in electromagnetic separation processes.

Among the several proposed schemes, the AC magnetic field method by applying an AC current in a solenoid was found to have the least dependence of the removal efficiency upon the particle size, which makes it most favorable for the separation of small inclusions. ${ }^{15}$ Experimental results ${ }^{11)}$ show that the electromagnetic separation is effective even in the case with strong electromagnetic stirring for this scheme. Although a stationary liquid model and a complete mixing model $^{9)}$ were presented to calculate the separation efficiency of inclusions, effects of the induced flow on the particle behavior and the overall separation efficiency are yet to be investigated in such separators. In the present study, continuous separation of inclusions from aluminum melt flowing in a square channel by applying an AC current in a solenoid is analyzed theoretically. Taniguchi and Brimacombe's model ${ }^{8)}$ is used to calculate the secondary flow of the melt and the separation efficiency of inclusions. Effects of various operating parameters that affect the secondary flow and the separation efficiency are discussed. Trajectories of a single inclusion particle in the melt are also calculated numerically to account for the effects of secondary flow on the separation efficiency. Finally, experimental work that has been done to verify the computed results of the separation efficiency will be described.

\section{Computational Method}

\subsection{Simplifying Assumptions}

As shown in Fig. 1, a liquid metal containing suspended inclusion particles is passed through an infinitely long square channel with a constant velocity, $w$, in the axial direction of the channel. The alternating magnetic field is generated by applying an alternating current in a solenoid coil. The following assumptions were made:

(1) The fluid flow is laminar and in steady state;

(2) The fluid flow is not affected by the movement of the particles;

(3) The electromagnetic field is not affected by the fluid flow;

(4) The inclusion particle is assumed as a non-conducting solid sphere;

(5) The interaction between the inclusion particles is neglected;

(6) The particle ceases to move when it reaches the wall of the pipe.

\subsection{Electromagnetic Force Field}

Suppose the electromagnetic field inside the channel is time harmonic field and does not vary with the axial distance, the governing equation of magnetic field strength, $H$, is expressed by the following equation:

$$
\frac{\partial^{2} H}{\partial x^{2}}+\frac{\partial^{2} H}{\partial y^{2}}=\alpha^{2} H
$$

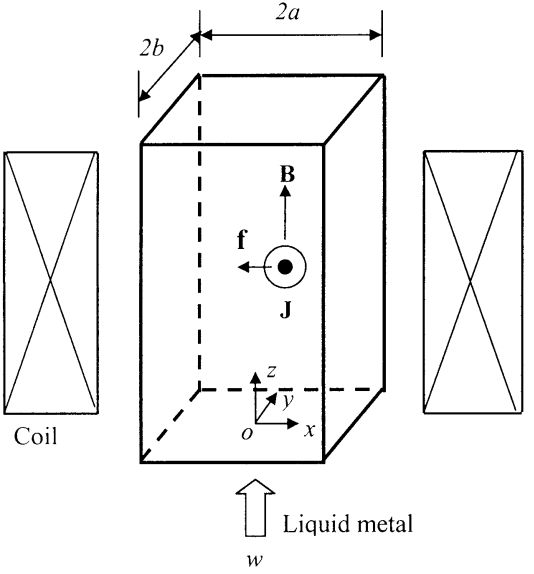

Fig. 1. Continuous separation of inclusions from aluminum melt flowing in a square channel.

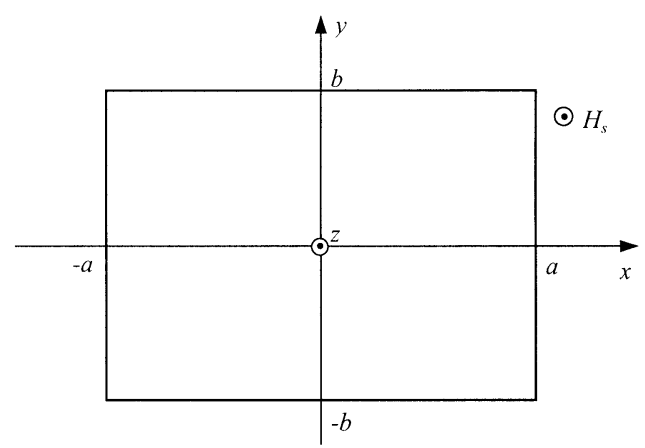

Fig. 2. The electromagnetic field inside the square channel.

where, $a=(1+j) / \delta, j=\sqrt{-1}$, skin depth $\delta=\sqrt{2 / \mu_{\mathrm{e}} \sigma \omega}$, angular frequency $\omega=2 \pi f, f$ is the frequency of magnetic field, $\mu_{\mathrm{e}}$ and $\sigma$ are magnetic permeability and electrical conductivity of liquid metal, respectively.

As shown in Fig. 2, the boundary conditions are expressed by:

$$
H=H_{\mathrm{s}} e^{j \omega t}, \quad x= \pm a \text { or } y= \pm b .
$$

where $H_{\mathrm{s}}=\sqrt{2} B_{\mathrm{e}} / \mu_{\mathrm{e}}$, is the amplitude of the imposed phasor magnetic field strength inside the coil, and $B_{\mathrm{e}}$ is the imposed effective magnetic flux density.

According to Ref. 16), the solution to Eqs. (1) and (2) is as follows:

$$
\begin{array}{r}
H=H_{s} \frac{\cosh \alpha y}{\cosh \alpha b}+\sum_{n=1,3,5}^{\infty} Q_{n} \cos \frac{n \pi y}{2 b} \cosh \sqrt{\left(\alpha^{2}+\frac{n^{2} \pi^{2}}{4 b^{2}}\right)} x \\
Q_{n}=\frac{\frac{4 \alpha^{2}}{n \pi} \sin \frac{n \pi}{2}}{\alpha^{2}+\frac{n^{2} \pi^{2}}{4 b^{2}}} \cdot \frac{H_{s}}{\cosh \sqrt{\left(\alpha^{2}+\frac{n^{2} \pi^{2}}{4 b^{2}}\right)} \ldots \ldots \ldots \ldots \ldots \ldots \ldots \ldots \ldots \ldots \ldots \ldots \ldots \ldots \ldots \ldots \ldots \ldots \ldots \ldots \ldots \ldots \ldots \ldots \ldots \ldots \ldots \ldots \ldots \ldots} \ldots
\end{array}
$$

The induced electric current density in the liquid metal can be obtained from Ampere's law as:

$$
\begin{gathered}
J_{x}=\frac{\partial H}{\partial y} \\
J_{y}=-\frac{\partial H}{\partial x}
\end{gathered}
$$


where $H, J_{x}$, and $J_{y}$ are time-independent part of respective corresponding time-varying variables.

Then the components of time-averaged electromagnetic force density, $f_{x}$ and $f_{y}$, are given by:

$$
\begin{gathered}
f_{x}=\frac{1}{2} \operatorname{Re}\left\{J_{y} \cdot \mu_{\mathrm{e}} H^{*}\right\} \\
f_{y}=-\frac{1}{2} \operatorname{Re}\left\{J_{x} \cdot \mu_{\mathrm{e}} H^{*}\right\}
\end{gathered}
$$

where $R e$ is the real part of the complex number and $H^{*}$ is the conjugate of the complex number, $H$.

\subsection{Flow Field}

The mathematical description of flow field is represented by the continuity equation and the Navier-Stokes equations. Suppose the fluid velocities do not change with the axial distance, the governing equations take the following form:

$$
\begin{aligned}
& \frac{\partial u}{\partial x}+\frac{\partial v}{\partial y}=0 \\
& \rho_{\mathrm{f}} u \frac{\partial u}{\partial x}+\rho_{\mathrm{f}} v \frac{\partial u}{\partial y}=-\frac{\partial p}{\partial x}+f_{x}+\mu_{\mathrm{f}}\left(\frac{\partial^{2} u}{\partial x^{2}}+\frac{\partial^{2} u}{\partial y^{2}}\right) \\
& \rho_{\mathrm{f}} u \frac{\partial v}{\partial x}+\rho_{\mathrm{f}} v \frac{\partial v}{\partial y}=-\frac{\partial p}{\partial x}+f_{y}+\mu_{\mathrm{f}}\left(\frac{\partial^{2} v}{\partial x^{2}}+\frac{\partial^{2} v}{\partial y^{2}}\right) \\
& \rho_{\mathrm{f}} u \frac{\partial w}{\partial x}+\rho_{\mathrm{f}} v \frac{\partial w}{\partial y}=-\frac{\partial p}{\partial z}+\mu_{\mathrm{f}}\left(\frac{\partial^{2} w}{\partial x^{2}}+\frac{\partial^{2} w}{\partial y^{2}}\right)
\end{aligned}
$$

where $u, v$, and $w$ are components of fluid velocities, $p$ is pressure, $\rho_{\mathrm{f}}$ and $\mu_{\mathrm{f}}$ are density and viscosity of liquid metal respectively. The $f_{x}$ and $f_{y}$ terms are the components of the electromagnetic force density, Eqs. (7) and (8).

The domain of calculation was restricted to a quarter cross section $(0 \leq x \leq a, 0 \leq y \leq b)$ and was divided into $25 \times$ 25 uniform meshes in the $x-y$ plane. The boundary conditions are no-slip conditions at the wall and the following equations at the symmetric surfaces:

$$
\begin{cases}x=0,0<y<b: & u=\frac{\partial v}{\partial x}=\frac{\partial w}{\partial x}=0 \\ y=0,0<x<a: & v=\frac{\partial u}{\partial y}=\frac{\partial w}{\partial y}=0\end{cases}
$$

The computational scheme employed in the present study is based on the finite volume method. The central differencing approximation was used to represent the diffusion terms, and the power-law scheme was used to approximate the convection terms. The SIMPLER algorithm ${ }^{17)}$ was adopted for the calculation of the flow field.

The solution procedure is analogous to Ref. 8). Eqations (9), (10) and (11) were solved first to give the velocity components and pressure distribution in the cross section. Then Eq. (12) was solved to compute the axial component of velocities at the given axial pressure gradient, $\partial p / \partial z$.

\subsection{Separation Efficiency of Inclusions}

The separation efficiency of inclusions is obtained by using the particle-concentration method, where the calcula- tion of particle concentrations is based on the following equation: ${ }^{8)}$

$$
\frac{\partial u_{\mathrm{p}} C}{\partial x}+\frac{\partial v_{\mathrm{p}} C}{\partial y}+\frac{\partial w_{\mathrm{p}} C}{\partial z}
$$

in which $C=c / c_{0}$, is the dimensionless particle concentration, $c_{0}$ is the particle concentration at the inlet of the square channel, and the migration velocity components of the particles, $u_{\mathrm{p}}, v_{\mathrm{p}}$ and $w_{\mathrm{p}}$, are expressed by: ${ }^{8)}$

$$
\begin{array}{r}
u_{\mathrm{p}}=u-\frac{d_{\mathrm{p}}^{2}}{24 \mu_{\mathrm{f}}} f_{x} \\
v_{\mathrm{p}}=v-\frac{d_{\mathrm{p}}^{2}}{24 \mu_{\mathrm{f}}} f_{y} \\
w_{\mathrm{p}}=w \ldots \ldots \ldots
\end{array}
$$

where $d_{\mathrm{p}}$ is the diameter of the particle. Note that the flotation velocity due to the buoyancy force is neglected in Eq. (17), because most oxide inclusions suspended in molten aluminum are very similar to the melt in density. ${ }^{18)}$

Equation (14) has been transformed into finite difference equations by the upwind differencing scheme. Because the convection flux of particles across a cell face is dependent on the particle concentration of its donor cell, the particle concentrations at location $z+\Delta z$ can be calculated based on the concentration values at the location $z$ for each step of $\Delta z$ along the flow direction, $z$. All the particle concentrations in each cell are obtained simultaneously by solving the discretised equations using the SOR method.

The separation efficiency of inclusions are evaluated by comparing the particle concentrations between the different cross sections, which takes the following form:

$$
\eta=1-\frac{\int_{0}^{a} \int_{0}^{b} w C d x d y}{\bar{w} a b}
$$

in which the average axial velocity, $\bar{w}$, can be obtained from:

$$
\bar{w}=\frac{\int_{0}^{a} \int_{0}^{b} w d x d y}{a b}
$$

\subsection{Trajectory of an Inclusion Particle}

Suppose the inclusion particle responds to the time-averaged force and the density difference between the particle and the melt is ignored, the motion of particles can be described by the following equation:

$$
\left(\rho_{\mathrm{p}}+C_{\mathrm{A}} \rho_{\mathrm{f}}\right) \frac{\pi d_{\mathrm{p}}^{3}}{6} \frac{d \mathbf{v}_{\mathrm{p}}}{d t}=\rho_{\mathrm{f}} C_{D} \frac{\pi d_{\mathrm{p}}^{2}}{8}\left|\mathbf{v}-\mathbf{v}_{\mathrm{p}}\right|\left(\mathbf{v}-\mathbf{v}_{\mathrm{p}}\right)-\frac{3}{4} \mathbf{f} \frac{\pi d_{\mathrm{p}}^{3}}{6}
$$

where the virtual mass coefficient $C_{\mathrm{A}}=0.5, \rho_{\mathrm{p}}$ is the density of the particle, and $\mathbf{v}$ and $\mathbf{v}_{\mathrm{p}}$ are the instantaneous velocity of the melt and the particle, respectively. The first term on the right-hand of the equation represents the viscous drag force on the particle, where the drag coefficient, $C_{\mathrm{d}}$, may be calculated by using the following equation: ${ }^{19}$ 


$$
C_{\mathrm{d}}=\frac{24}{\operatorname{Re}_{\mathrm{d}}}\left(1+0.15 \mathrm{Re}_{\mathrm{d}}^{0.687}\right) \quad \text { for } \quad \mathrm{Re}_{\mathrm{d}} \leq 1000
$$

in which the particle Reynolds number is defined as:

$$
\operatorname{Re}_{\mathrm{d}}=\frac{\rho_{\mathrm{f}} d_{\mathrm{p}}\left|\mathbf{v}-\mathbf{v}_{\mathrm{p}}\right|}{\mu_{\mathrm{f}}}
$$

It should be pointed out that the Basset history force term is neglected in Eq. (20) for the sake of simplicity. However, the term associated with the electromagnetic expulsive force exerted on the particle is included directly rather than the pressure gradient term used in El-Kaddah's model. ${ }^{14)}$

Equation (20) was solved numerically using the adaptive Gill method, and the time step is specified as $\Delta t=0.0001 \mathrm{~s}$. The solutions thus obtained give the instantaneous particle velocity. Then the particle position in the cross section of the melt in the next time step may be calculated using the following equation:

$$
\left\{\begin{array}{l}
x=x_{0}+u_{\mathrm{p}} \Delta t \\
y=y_{0}+v_{\mathrm{p}} \Delta t
\end{array}\right.
$$

with the initial conditions specified as:

$$
t=0: x=x_{0}, y=y_{0}, u_{\mathrm{p}}=u, v_{\mathrm{p}}=v
$$

The particle trajectory in the cross section may be determined by iterating the above solution procedure until the particle reaches the wall of the channel.

\section{Experimental Procedure}

The experimental apparatus for continuous separation of non-metallic inclusions from aluminum melt is composed of an induction coil, a multi-channel separator and a gating system as sketched in Fig. 3. The induction coil is made up of copper tubes. The inner diameter of the coil is $60 \mathrm{~mm}$ and its length is $120 \mathrm{~mm}$. The coil is powered by one IGBTtype induction heating power supply, which is capable of producing the alternating electric currents with a frequency of $8.3 \mathrm{kHz}$ or $15.6 \mathrm{kHz}$. The separator used in experiments is a sintered gypsum pipe with four open square channels in its cross section. The cross-sectional size of each channel is $10 \mathrm{~mm} \times 10 \mathrm{~mm}$.

The alloy used in experiments is prepared by melting pure aluminum with $\mathrm{Al} 5 \% \mathrm{Si}-6 \% \mathrm{Al}_{2} \mathrm{O}_{3}$ particle reinforced composites. The final composition of the alloy is $3-4 \% \mathrm{Si}$, $1.5-2.5 \% \mathrm{Al}_{2} \mathrm{O}_{3}$ with the balance $\mathrm{Al}$. The measured average diameter of the particles for preparation of the composites is found to be $6 \mu \mathrm{m}$ and most of the particles are in the range of $3-12 \mu \mathrm{m}$ in diameter.

For each experiment, about $1.5-\mathrm{kg}$ alloy contained in a graphite crucible was melted and kept at $800^{\circ} \mathrm{C}$ for $5 \mathrm{~min}$ in a resistance furnace. After the IGBT power supply was on, the melt was poured into the pouring cup and flowed continuously through the separator channels. The pouring rate is controlled by maintaining the metal head in the pouring cup and the average flow rate of the melt through the separator is calculated by weighting the processed metal in a certain time period.

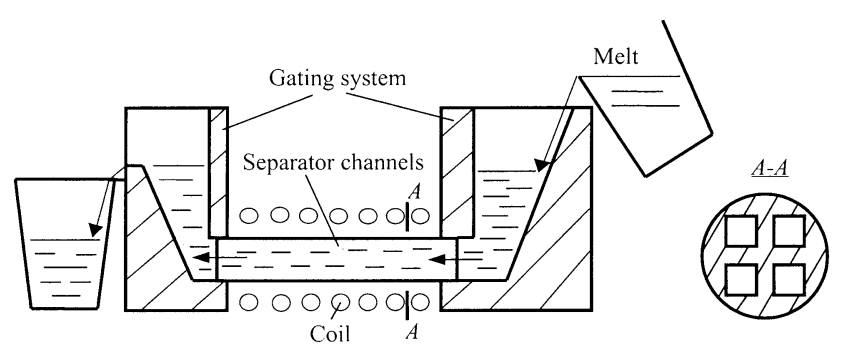

Fig. 3. Experimental apparatus for continuous separation of nonmetallic inclusions from aluminum melt.

Table 1. Property values of alumina particles-molten aluminum system used for calculations.

\begin{tabular}{lcccc}
\hline & Magnetic & Electrical & Density & Viscosity \\
& Permeability & Conductivity & $\left(\mathrm{kg} / \mathrm{m}^{3}\right)$ & $(\mathrm{Pa} \cdot \mathrm{s})$ \\
& $(\mathrm{H} / \mathrm{m})$ & $(\mathrm{S} / \mathrm{m})$ & & \\
\hline Liquid metal & $4 \pi \times 10^{-7}$ & $2.95 \times 10^{6}$ & $2.37 \times 10^{3}$ & $2.5 \times 10^{-3}$ \\
Inclusion particle & - & - & $3.99 \times 10^{3}$ & - \\
\hline
\end{tabular}

Samples before and after processing were taken to evaluate the separation efficiency of inclusions by the quantitative metallograph method. The total area of inclusions in 25-50 fields of view for each sample was measured by a computerized image analysis system and the separation efficiency, $\eta$, is represented by

$$
\eta=\left(1-\frac{S_{1}}{S_{0}}\right) \times 100 \%
$$

where, $S_{0}$ and $S_{1}$ are the measured area of the inclusions before and after processing, respectively.

\section{Results and Discussion}

Table 1 gives some property values associated with the alumina particles-molten aluminum system for the following calculations.

\subsection{Electromagnetic Force Field}

Figure 4 shows the computed electromagnetic force field in a quarter cross section of the square channel. It is seen that the liquid metal is subjected to electromagnetic pinch force which is directed towards the center of the channel. It is also seen that the distribution of electromagnetic force density in the melt is significantly uneven. Due to the skin effect of high frequency magnetic field, the electromagnetic force is very weak in the central region of the channel. Another region where the electromagnetic force is relatively weak is near the corner, while the electromagnetic force is almost very strong in the outer region.

\subsection{Flow Field}

Due to the nonhomogeneous electromagnetic force field, there will appear induced secondary flow in the cross section other than the axial flow. Figure 5 gives the computed velocity field in a quarter cross section of the channel. It is seen that there are two recirculating loop flows in a quarter cross section of the channel. Consistent with the electro- 


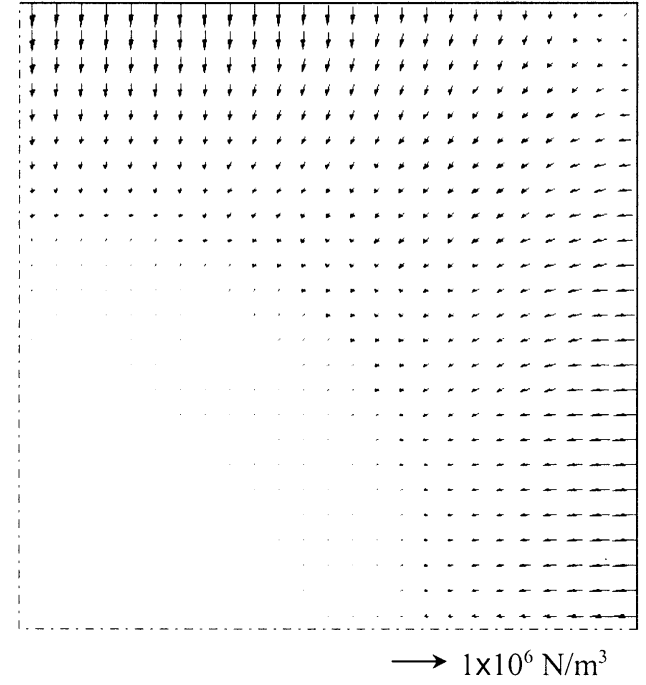

Fig. 4. Vector diagram of the electromagnetic force density in a quarter cross section of the square channel $\left(B_{\mathrm{e}}=0.04 \mathrm{~T}\right.$, $f=13.692 \mathrm{kHz}, a=b=5 \mathrm{~mm})$.

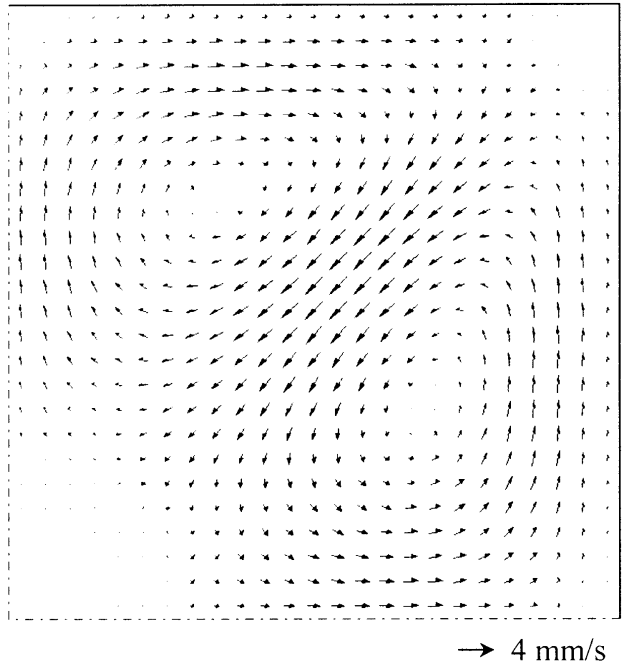

Fig. 5. Computed velocity field in a quarter cross section of the square channel $\left(B_{\mathrm{e}}=0.04 \mathrm{~T}, f=13.692 \mathrm{kHz}, a=b=5 \mathrm{~mm}\right)$.

magnetic force field shown in Fig. 4, the secondary flow is negligible in the central region and near the corner. However, the scope of the central region where the secondary flow is insignificant is greatly confined as compared with the region within which the electromagnetic force is very weak.

Despite the stirred melt, the pressure gradient generated in the melt seems unaffected and remains remarkable, especially in the outer region, as shown in Fig. 6.

Besides, the calculated axial velocity is found to be decreased with the development of secondary flow because of the increase in the flow resistance.

\subsection{Effects of Secondary Flow on Separation Ef- ficiency}

Figure 7 gives the computed separation efficiency of 5, 10 , and $20 \mu \mathrm{m}$ inclusions as a function of $t_{i}$, the average resident time of inclusions within the $z_{i}$ length of the channel which is defined as $t_{i}=z_{i} / \bar{w}$. The solid lines and dashed lines represent the separation efficiency of inclusions with and without taking the secondary flow into consideration,

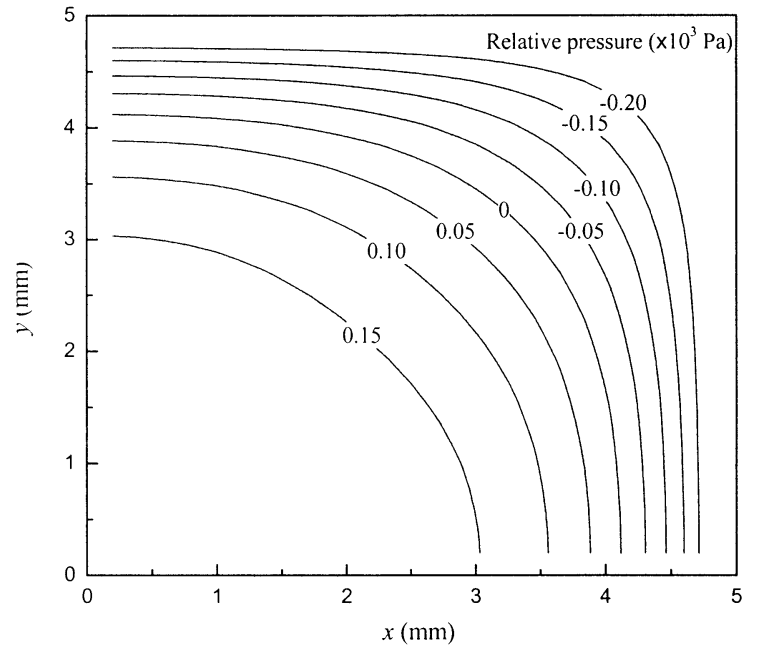

Fig. 6. Computed pressure field in a quarter cross section of the channel $\left(B_{\mathrm{e}}=0.04 \mathrm{~T}, f=13.692 \mathrm{kHz}, a=b=5 \mathrm{~mm}\right)$.

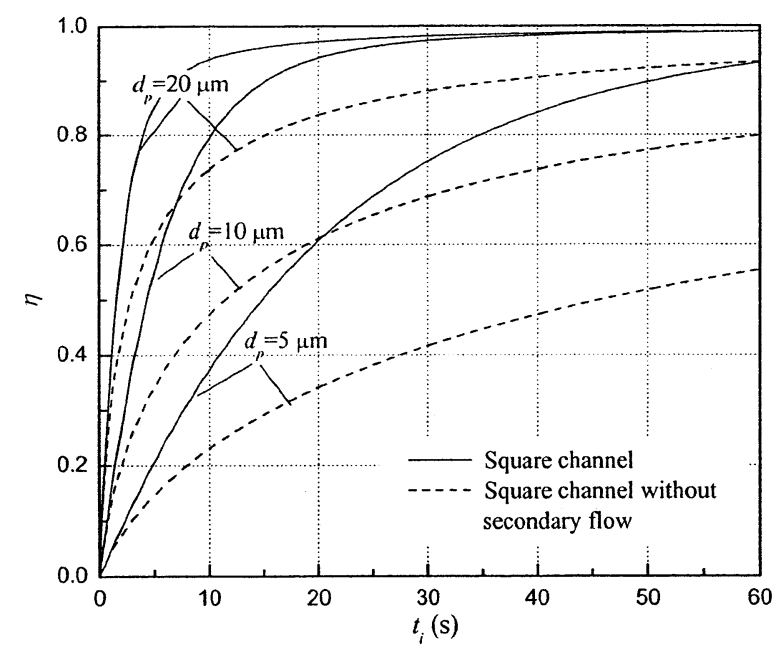

Fig. 7. Effects of secondary flow on the separation efficiency $\left(B_{\mathrm{e}}=0.04 \mathrm{~T}, f=13.692 \mathrm{kHz}, a=b=5 \mathrm{~mm}, \partial p / \partial z=-10\right.$ $\left.\mathrm{N} / \mathrm{m}^{3}\right)$.

respectively. It is seen that the separation efficiency of inclusions is greatly increased due to the secondary flow.

Figures 8(a) and 8(b) show the concentration profiles of the inclusion particles with and without taking the secondary flow into consideration, respectively. It is seen that the secondary flow has a mixing effect on the distribution of the inclusions that significantly diminishes the difference in particle concentrations between the inner region and outer region in the cross section of the square channel.

Besides the mixing effect in the cross section, the secondary flow influences the axial velocities of liquid metal as well. The decrease in the axial velocities leads to the increase of the average resident time of inclusions and may also affect the distribution of inclusion particles, which is shown in Fig. 8(c), where the mixing effect is eliminated by setting the $x$ - and $y$-components of fluid velocity to zero in calculations. It is seen that the change in the axial velocities caused by the secondary flow has a negligible effect on the particle concentrations and separation efficiency as compared with the mixing effect. Consequently, the main reason for the increase of the separation efficiency is owing to the mixing effect of the secondary flow. 


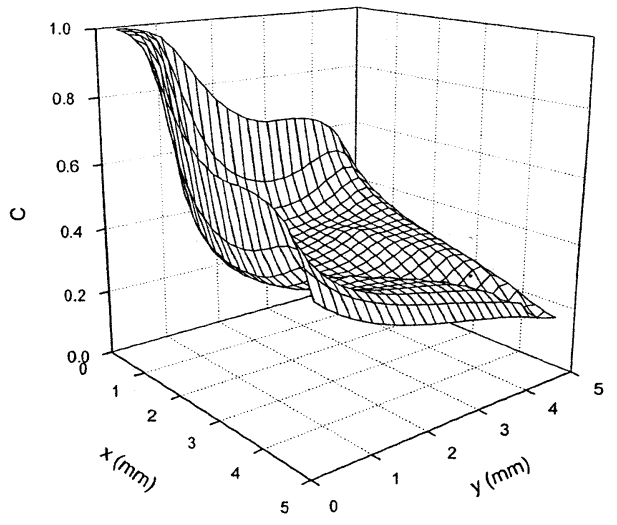

(a) with the secondary flow, $\eta=0.552$

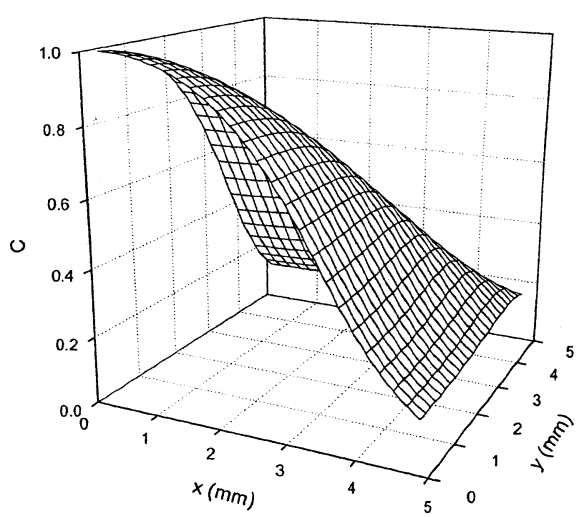

(b) without the secondary flow, $\eta=0.341$

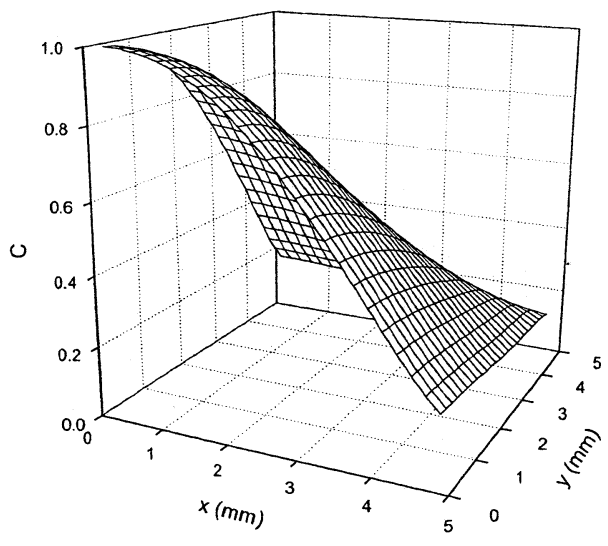

(c) without the mixing effect, $\eta=0.380$
Fig. 8. Particle concentration map in a quarter cross section of the square channel $\left(d_{\mathrm{p}}=10 \mu \mathrm{m}, t_{i}=5 \mathrm{~s}, B_{\mathrm{e}}=\right.$ $0.04 \mathrm{~T}, f=13.692 \mathrm{kHz}, a=b=5 \mathrm{~mm}$, $\left.\partial p / \partial z=-10 \mathrm{~N} / \mathrm{m}^{3}\right)$.

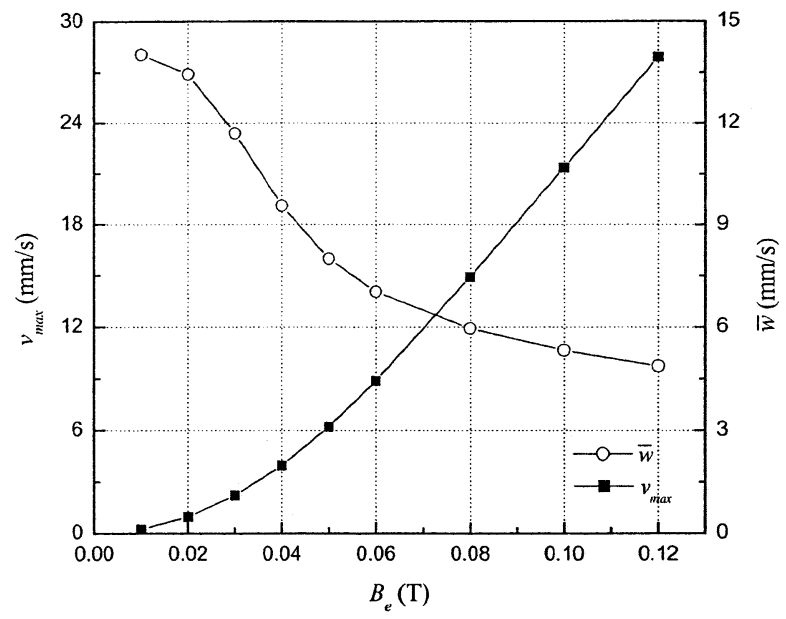

Fig. 9. Variation of $v_{\max }$ and $\bar{w}$ with the effective magnetic flux density $\left(f=13.692 \mathrm{kHz}, a=b=5 \mathrm{~mm}, \partial p / \partial z=-10 \mathrm{~N} / \mathrm{m}^{3}\right)$.

\subsection{Effects of Various Operating Parameters on Separation Efficiency}

Various operating parameters may affect the secondary flow and the separation efficiency, including the effective magnetic flux density, the frequency of magnetic field, and the size of the separator channels.

\subsubsection{Effective Magnetic Flux Density}

The effects of the effective magnetic flux density, $B_{\mathrm{e}}$, on the secondary flow are given in Fig. 9, where the maximum

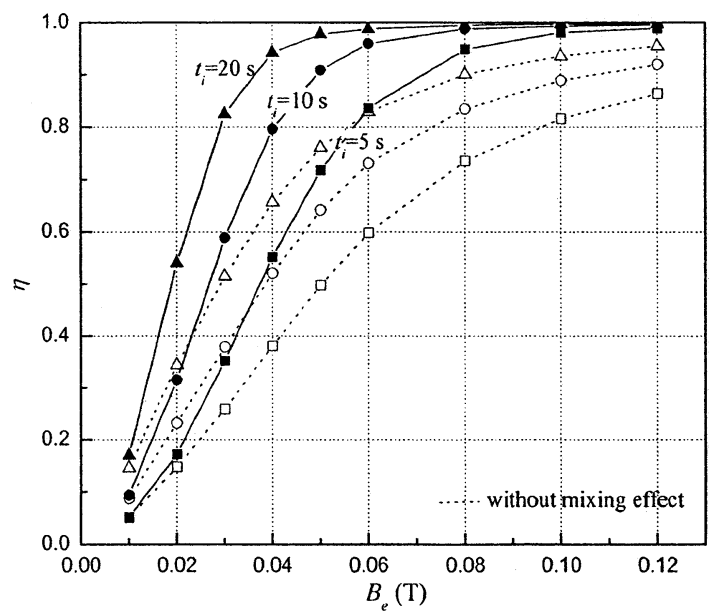

Fig. 10. Relation between the separation efficiency and the effective magnetic flux density $\left(d_{\mathrm{p}}=10 \mu \mathrm{m}, f=13.692 \mathrm{kHz}\right.$, $\left.a=b=5 \mathrm{~mm}, \partial p / \partial z=-10 \mathrm{~N} / \mathrm{m}^{3}\right)$.

velocity inside the cross section, $v_{\max }$, reflects the intensity of the secondary flow. It is seen that the intensity of the secondary flow increases markedly with the increase of $B_{\mathrm{e}}$, and the average axial velocity decreases with the development of the secondary flow.

Figure 10 shows the relation between the separation efficiency and the effective magnetic flux density. It is seen that the separation efficiency increases with the increase of the effective magnetic flux density and the improvement of 


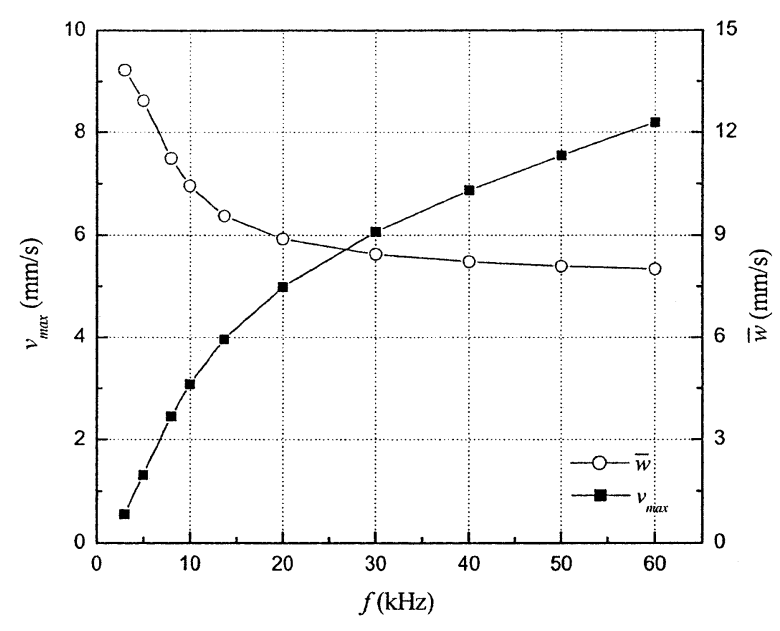

Fig. 11. Variation of $v_{\max }$ and $\bar{w}$ with the frequency of the magnetic field $\left(B_{\mathrm{e}}=0.04 \mathrm{~T}, a=b=5 \mathrm{~mm}, \partial p / \partial z=-10 \mathrm{~N} / \mathrm{m}^{3}\right)$.

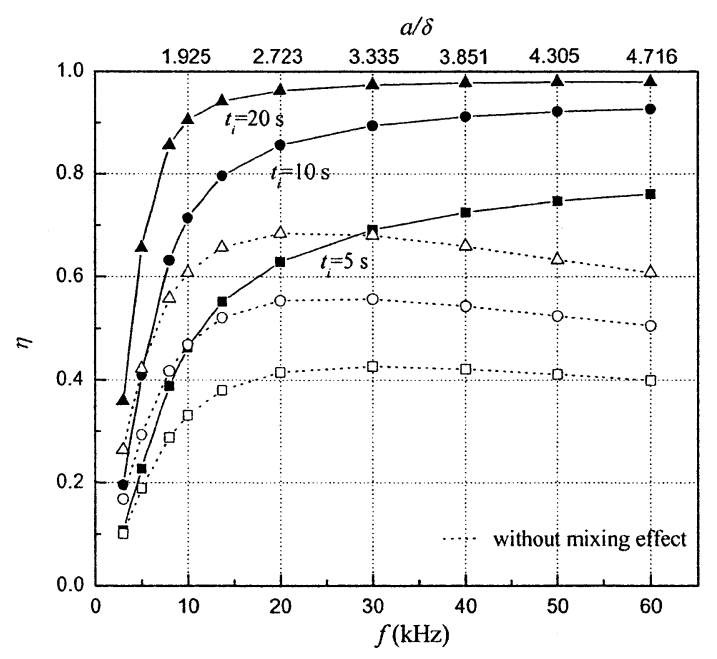

Fig. 12. Relation between the separation efficiency and the frequency of the magnetic field $\left(d_{\mathrm{p}}=10 \mu \mathrm{m}, B_{\mathrm{e}}=0.04 \mathrm{~T}\right.$, $\left.a=b=5 \mathrm{~mm}, \partial p / \partial z=-10 \mathrm{~N} / \mathrm{m}^{3}\right)$.

the separation efficiency due to the mixing effect becomes more significant with increasing $B_{\mathrm{e}}$.

\subsubsection{Frequency of the Magnetic Field}

The effects of the frequency of the magnetic field, $f$, on the secondary flow are shown in Fig. 11. It is seen that the secondary flow is stronger and the average axial velocity is smaller with increasing $f$.

Figure 12 shows the effects of the frequency of the magnetic field on the separation efficiency. If the mixing effect is not considered, as known in Refs. 9) and 15), the separation efficiency reaches the maximum values at about $a / \delta=2$, and drops with further increase of the frequency due to more remarkable skin effect of the magnetic field which limits the elimination of the inclusions in the inner region. When the effects of secondary flow are taken into account, it is seen that the separation efficiency is greatly increased. Especially, even for higher frequency of the magnetic field that $a / \delta>2$, the mixing effect of secondary flow is still strong enough to compensate the decrease of the separation efficiency caused by the skin effect of the magnetic field.

\subsubsection{Size of the Separator Channel}

The size of the separator channel affects the secondary

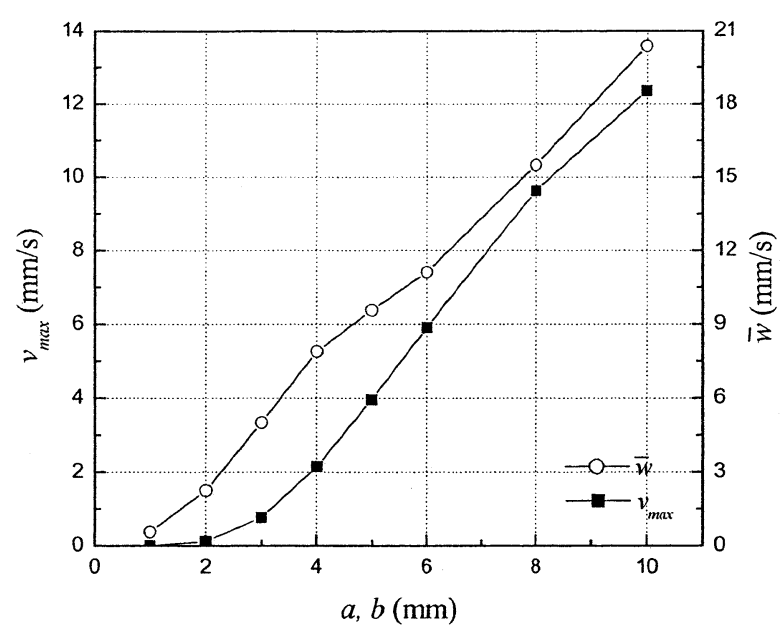

Fig. 13. Variation of $v_{\max }$ and $\bar{w}$ with the size of the separator $\left(B_{\mathrm{e}}=0.04 \mathrm{~T}, f=13.692 \mathrm{kHz}, \partial p / \partial z=-10 \mathrm{~N} / \mathrm{m}^{3}\right)$.

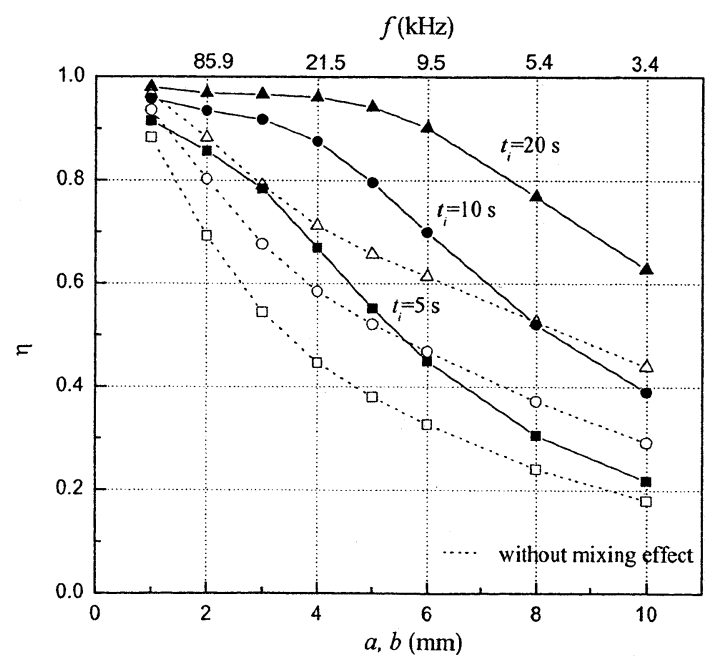

Fig. 14. Relation between the separation efficiency and the size of the separator channel at constant $a / \delta(=2)\left(d_{\mathrm{p}}=10\right.$ $\left.\mu \mathrm{m}, B_{\mathrm{e}}=0.04 \mathrm{~T}, \partial p / \partial z=-10 \mathrm{~N} / \mathrm{m}^{3}\right)$.

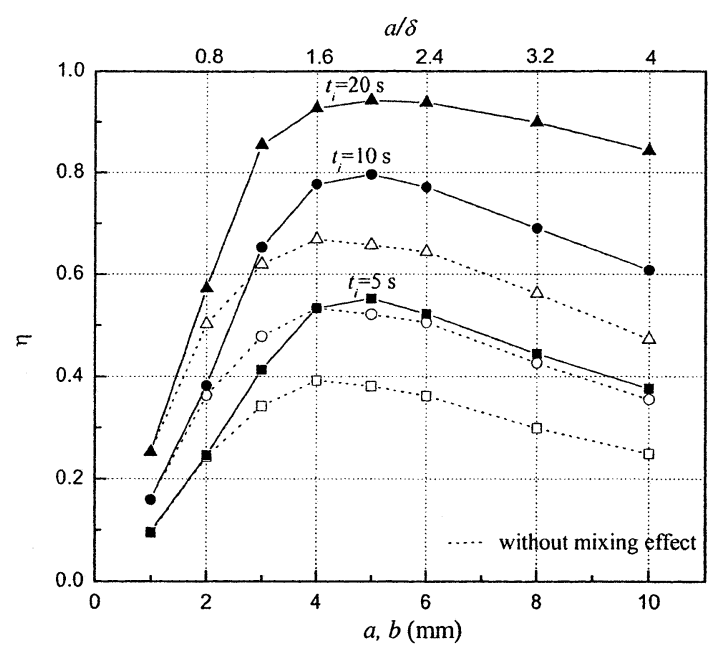

Fig. 15. Relation between the separation efficiency and the size of the separator channel at constant frequency $f=$ $13.692 \mathrm{kHz}\left(d_{\mathrm{p}}=10 \mu \mathrm{m}, B_{\mathrm{e}}=0.04 \mathrm{~T}, \partial p / \partial z=-10 \mathrm{~N} / \mathrm{m}^{3}\right)$.

flow considerably. As shown in Fig. 13, both $v_{\max }$ and $\bar{w}$ increase with the increase of the side length of the square channel, which indicates that the use of thin channels may suppress the flow of melt.

Figure 14 shows the effects of the size of the separator 
channel on the separation efficiency at a constant $a / \delta$. It is seen that the separation efficiency decreases significantly with the increase of the size of the separator channels despite the mixing effect of the secondary flow. This is mainly because that the particles have to migrate across a longer distance before arriving at the wall.

Figure 15 gives the relation between the separation efficiency and the size of the channel when the frequency of the magnetic field is kept constant. It is seen that, although the separation efficiency reaches the maximum values at about $a / \delta=2$, it still maintains a considerable value for a larger size of the channel due to the increasing secondary flow.

From the calculations, it is possible to achieve high separation efficiency of inclusions with the help of the mixing effect of secondary flow by using large-sized separator channels and magnetic field of high frequency. The significance of the idea is that it provides a way to achieve high removal efficiency of inclusions in large volumes of melt without securing homogeneous distribution of electromagnetic force, which is helpful to the implementation of electromagnetic separation technique in industrial applications.

\subsection{Trajectories of an Inclusion Particle}

In this section, trajectories of a single inclusion particle in a quarter cross section of the channel are calculated in order to account for the effects of secondary flow on the separation efficiency. Figure 16 computes the particle trajectories at the different initial locations as well as the separation time, where the dotted lines show the particle trajec-

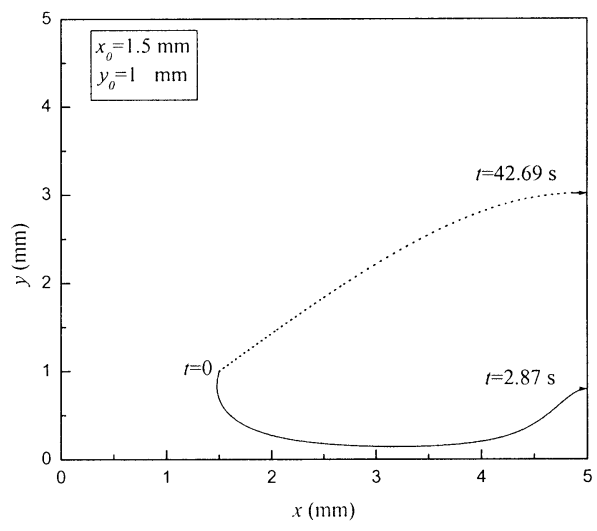

(a) $x_{0}=1.5 \mathrm{~mm}, y_{0}=1 \mathrm{~mm}$

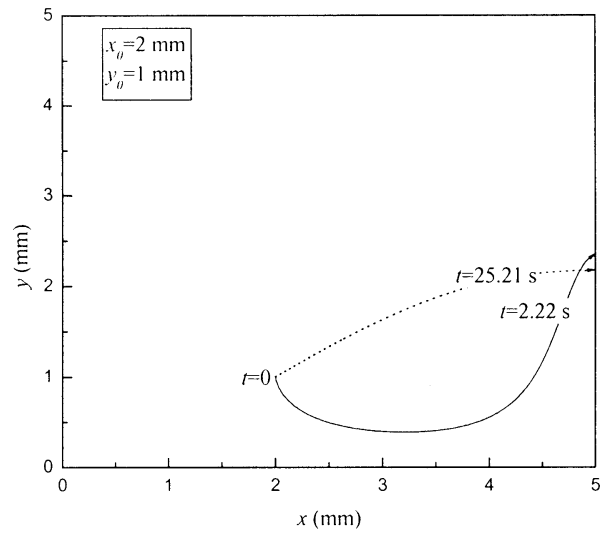

(b) $x_{0}=2 \mathrm{~mm}, y_{0}=1 \mathrm{~mm}$

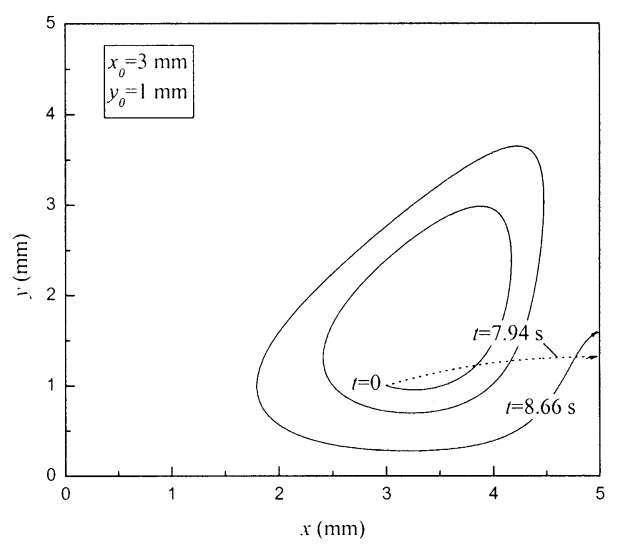

(d) $x_{0}=3 \mathrm{~mm}, y_{0}=1 \mathrm{~mm}$

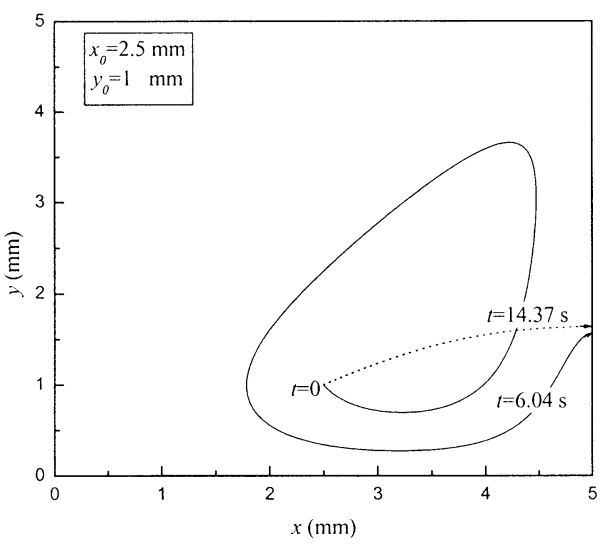

(c) $x_{0}=2.5 \mathrm{~mm}, y_{0}=1 \mathrm{~mm}$

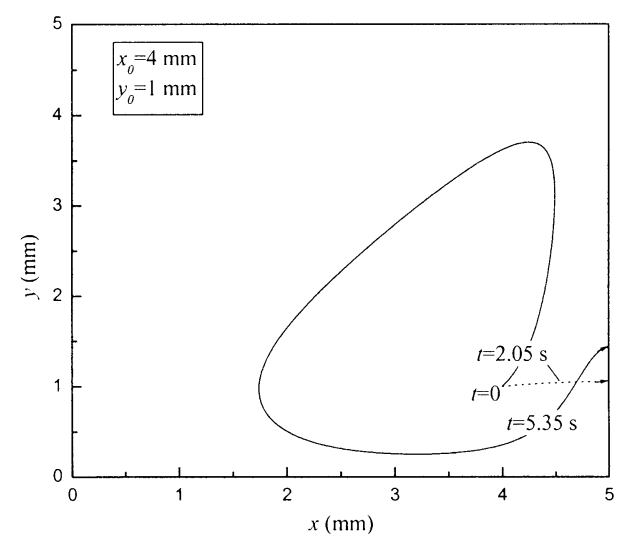

(e) $x_{0}=4 \mathrm{~mm}, y_{0}=1 \mathrm{~mm}$
Fig. 16. Effects of secondary flow on the separation time of inclusions $\left(d_{\mathrm{p}}=10 \mu \mathrm{m}, B_{\mathrm{e}}=0.04 \mathrm{~T}\right.$, $f=13.692 \mathrm{kHz}, \quad a=b=5 \mathrm{~mm}$, $\left.\partial p / \partial z=-10 \mathrm{~N} / \mathrm{m}^{3}\right)$. 
tories without taking the secondary flows into consideration. It is seen that the secondary flow accelerates the transportation of the particles from the inner region to the vicinity of the wall and greatly shorten the separation time of those particles, as shown in Figs. 16(a)-16(c), while it may cause the entrapment of the particles in the outer region within the flow eddies and thus delay the separation, which is shown in Figs. 16(d) and 16(e). As a result, the exchange of the particles between the inner region and the outer region is enhanced with the circulating convection flows, which may account for the mixing effect of the secondary flow on the distribution of inclusions.

As shown in Fig. 4, the electromagnetic force is very weak in the inner region due to the skin effect of the high frequency magnetic field. Consequently, the particles located in this region cannot move to the wall efficiently if driven by the electromagnetic expulsive force only. Therefore, efficient separation of the particles in the inner region is central to the separation efficiency of the whole system. From the calculated results, the secondary flow just compensates the weakness of the electromagnetic effect in such region by transporting the particles with the convection flows from the inner region to the vicinity of the wall where strong electromagnetic force exists, and thus improves the separation efficiency of inclusions on the whole.

\subsection{Experimental Results}

Metallograph observations show that the distribution of inclusions in each channel after processing is uniform for the multi-channel separator used in experiments. Figure 17 gives the cross-sectional metallographs of the alloy remained in a square channel after processing. It can be observed that a large quantity of inclusions were captured by the wall near the inlet and in the middle of the channel, while fewer inclusions were seen at the outlet of the channel.

Table 2 summarizes the measured results of the separation efficiency for a square channel under different processing conditions, where $B_{\mathrm{e}}$ is the maximum measured value inside the coil, and $t_{i}$ is determined from the length of the coil and the average flow rate of the melt. The calculated values of the separation efficiency with and without considering the mixing effect of the secondary flow are also given in the table by using the corresponding values of the processing parameters. It is seen that the calculated separation efficiency with the mixing effect generally shows better agreement with the measured results than the calculated value without the mixing effect. Although a few important issues, such as the agglomeration of small particles within the flow eddies, the accumulation of particles on the wall and the axial variation of the magnetic flux density inside the coil, are not included to represent the actual experimental system completely, the theoretical values given by the computational method employed in the present study still clearly demonstrates the significant effects of the secondary flow on the separation efficiency and the potential of the electromagnetic separation technique by using large-sized square channels.

\section{Conclusions}

This study investigates the effects of secondary flow of the melt on the separation of inclusions from aluminum melt flowing in a square channel using an alternating magnetic field generated by a solenoid. Numerical methods have been used to calculate the secondary flow, the separation efficiency of inclusions, and the particle motion. It is found that there appear two recirculating loop flows in a

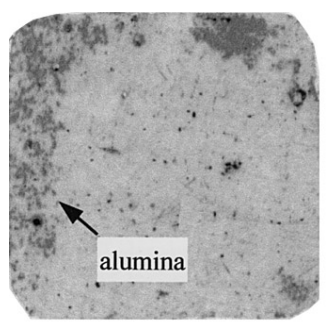

(a) near the inlet

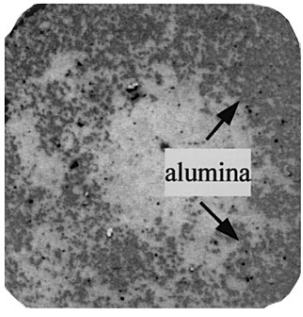

(b) in the middle

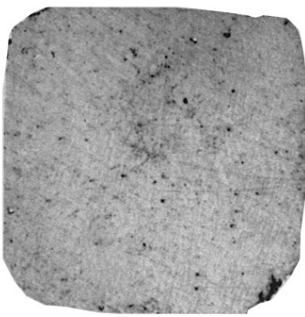

(c) at the outlet

Fig. 17. Cross-sectional metallographs of the alloy remained in a square separator channel $\left(t_{i}=22.6 \mathrm{~s}, B_{\mathrm{e}}=0.12 \mathrm{~T}\right.$, $f=15.6 \mathrm{kHz})$.

Table 2. Experimental and computed results of the separation efficiency under different processing conditions $\left(d_{\mathrm{p}}=6 \mu \mathrm{m}\right.$, $a=b=5 \mathrm{~mm})$.

\begin{tabular}{ccccccc}
\hline \multirow{2}{*}{$\begin{array}{c}\text { Experiment } \\
\text { No. }\end{array}$} & $B_{e}$ & $f$ & $t_{i}$ & Measured & \multicolumn{2}{c}{ Calculated separation efficiency (\%) } \\
\cline { 6 - 7 } & $(\mathrm{T})$ & $(\mathrm{kHz})$ & $(\mathrm{s})$ & separation & With the mixing & Without the \\
& & & & efficiency $(\%)$ & effect & mixing effect \\
\hline 1 & 0.085 & 8.3 & 16 & 76.5 & 95.2 & 71.0 \\
2 & 0.124 & 8.3 & 5 & 96.8 & 87.4 & 65.1 \\
3 & 0.043 & 15.6 & 9 & 69.6 & 53.8 & 34.6 \\
4 & 0.085 & 15.6 & 22.6 & 96.5 & 99.5 & 83.6 \\
\hline
\end{tabular}


quarter cross section of the channel due to the nonhomogeneous electromagnetic force field and the separation efficiency of inclusions is significantly improved by the secondary flow mainly owing to the mixing effect. The computed results of particle trajectories show that the secondary flow accelerates the transportation of the particles from the inner region to the vicinity of the wall and greatly shortens the separation time of those particles while it may cause the entrapment of the particles in the outer region within the flow eddies. Various operating parameters may affect the secondary flow as well as the separation efficiency, including the effective magnetic flux density, the frequency of magnetic field, and the size of the separator channel. It is found that the separation efficiency increases with the increase of the effective magnetic flux density and the frequency of magnetic field, and decreases significantly with the increase of the size of the separator channel for a constant value of $a / \delta$. However, it is possible to achieve high separation efficiency by using large-sized square channels and high frequency magnetic field with the help of the mixing effect of secondary flow. It is confirmed by comparing the measured separation efficiency with the computed results that the secondary flow improves the separation efficiency significantly.

\section{Acknowledgements}

This work was supported by the National Natural Science Foundation of China (Grant No. 59871029) and the National Key Fundamental Research Project (973) (No. G1999064900-4).

\section{Nomenclature}

$a, b:$ Size of a quarter cross section of square channel (m)

$B_{\mathrm{e}}$ : Effective magnetic flux density $(\mathrm{T})$

$C$ : Dimensionless particle concentration

$c:$ Particle concentration $\left(1 / \mathrm{m}^{3}\right)$

$d_{\mathrm{p}}$ : Particle diameter $(\mathrm{m})$

f: Electromagnetic force density $\left(\mathrm{N} / \mathrm{m}^{3}\right)$

$f$ : Frequency of magnetic field $(\mathrm{Hz})$

$H$ : Magnetic field strength $(\mathrm{A} / \mathrm{m})$

$J: \quad$ Induced electric current density $\left(\mathrm{A} / \mathrm{m}^{2}\right)$

$p$ : Pressure $(\mathrm{Pa})$

$t$ : Time (s)

$u: \quad x$-component of velocity $(\mathrm{m} / \mathrm{s})$

$\mathbf{v}$ : Velocity vector $(\mathrm{m} / \mathrm{s})$

$v: y$-component of velocity $(\mathrm{m} / \mathrm{s})$

$w: \quad z$-component of velocity $(\mathrm{m} / \mathrm{s})$

$\bar{w}$ : Average axial velocity of the melt $(\mathrm{m} / \mathrm{s})$ $v_{\max }:$ Maximum velocity of the melt in the cross section $(\mathrm{m} / \mathrm{s})$

$\delta: \quad$ Skin depth (m)

$\eta$ : Separation efficiency of inclusions

$\mu_{\mathrm{e}}$ : Magnetic permeability $(\mathrm{H} / \mathrm{m})$

$\mu_{\mathrm{f}}$ : Viscosity $(\mathrm{Pa} \cdot \mathrm{s})$

$\rho:$ Density $\left(\mathrm{kg} / \mathrm{m}^{3}\right)$

$\sigma:$ Electrical conductivity $(\mathrm{S} / \mathrm{m})$

$\omega$ : Angular frequency $(\mathrm{rad} / \mathrm{s})$

Subscripts

f: Fluid

$\mathrm{p}$ : Particle

$x: x$ direction

$y$ : $y$ direction

\section{REFERENCES}

1) N. El-Kaddah, A. D. Patel and T. T. Natarajan: JOM, 47 (1995), 46.

2) A. D. Patel and N. El-Kaddah: Proc. Light Metals 1997, TMS, Warrendale, PA, (1997), 1013.

3) D. Leenov and A. Kolin: J. Chem. Phys., 22 (1954), 683.

4) P. Marty and A. Alemany: Proc. Symp. of the IUTAM, Metallurgical Applications of Magnetohydrodynamics, Cambridge, UK, (1982), 245.

5) M. Garnier: Proc. of the 3rd Int. Symp. on Electromagnetic Processing of Materials, ISIJ, Tokyo, (2000), 3.

6) Kolin: Science, 117 (1953), 134.

7) J.-P. Park, A. Morihira, K. Sassa and S. Asai: Tetsu-to-Hagané, 80 (1994), 389.

8) S. Taniguchi and J. K. Brimacombe: ISIJ Int., 34 (1994), 722.

9) F. Yamao, K. Sassa, K. Iwai and S. Asai: Tetsu-to-Hagané, 83 (1997), 30

10) Y. Tanaka, K. Sassa, K. Iwai and S. Asai: Tetsu-to-Hagané, 81 (1995), 1120.

11) S. Taniguchi and A. Kikuchi: Proc. of the 3rd Int. Symp. on Electromagnetic Processing of Materials, ISIJ, Tokyo, (2000), 315.

12) S. Makarov, R. Ludwig and D. Apelian: IEEE Trans. Mag., 37 (2001), 1024.

13) S. Makarov, D. Apelian and R. Ludwig: AFS Trans., 107 (1999), 727.

14) N. El-Kaddah: IEEE on Industrial Applications, IEEE, Piscataway, NJ, (1988), 1162.

15) D. Shu, B. D. Sun, J. Wang, T. X. Li and Y. H. Zhou: Metall. Mater. Trans. A, 30 (1999), 2979.

16) R. L. Stoll: The Analysis of Eddy Currents, Oxford Clarendon Press, Oxford, (1974), 48.

17) S. V. Patankar: Numerical Heat Transfer and Fluid Flow, Hemisphere Publishing Corporation, Taylor \& Francis Group, New York, NY, (1980).

18) P. N. Crepeau: Mod. Cast., 87 (1997), 39.

19) G. B. Wallis: One-Dimensional Two-Phase Flow, McGraw-Hill, New York, NY, (1969), 178. 